\title{
Afecto y emoción en la ecología política urbana
}

\section{Affect and emotion in urban political ecology}

\author{
Jacob C. Miller
}

\begin{abstract}
Resumen
En los últimos 20 años, ha sido un giro hacia lo emocional y afectivo en la teoría social contemporánea y en varias partes de la geografía humana, incluyendo la ecología política urbana. Éste artículo ofrece una metodología para aproximar la emoción y el afecto en las geografías del consumo cotidiano, tomando los shopping malls como un lugar especial, por su enfoque en manipular esas áreas de la experiencia del visitante. Enfocando en un estudio etnográfico sobre el Mall Paseo Chiloé y su contexto de cambio socio-espacial rápido, éste artículo muestra algunos métodos para ese tipo de investigación y algunas reflexiones sobre mi experiencia usando estos métodos como una manera de mostrar que tipos de desafíos pueden aparecer. También están incluidos algunos resultados para mostrar el potencial de esa aproximación. Como ya existe literatura sobre los afectos y emociones en las ciencias sociales, este artículo se basa en el trabajo de campo y los aportes metodológicos para llevar a cabo tal estudio. Así, el énfasis está en los detalles alrededor de métodos fundamentales, como la observación participativa, métodos innovadores, como la entrevista móvil y algunas estrategias de usar materiales visuales en la investigación.
\end{abstract}

Palabras clave: afecto, consumo, Chiloé, emoción, metodología.

\begin{abstract}
In the last 20 years there has been a turn toward the emotional and affective in contemporary social theory and in human geography, including urban political ecology. This article offers a methodology for approximating emotion and affect in everyday geographies of consumption, taking the shopping mall as a special case due to its ability to manipulate these aspects of the visitor experience. Focusing on an ethnographic study of the Mall Paseo Chiloé and its context of rapid social-spatial change, this article describes the methods used for this kind of research, as well as offering some reflections on my experience using these methods as a way of highlighting the kinds of challenges that can arise during fieldwork. This article also includes some results from the fieldwork that highlight the potential of this methodology. As there is already a vast literatura on affect and emotion in the social sciences, this article focuses on the fieldwork and the methodological approach for carrying out such a study. As such, the emphasis is on the details around using fundamental methods like participant observation, as well as more innovative methods like mobile interviews and photo elicitation.
\end{abstract}

Keywords: affect, Chiloé, consumption, emotion, methodology.

Recibido el 5 de agosto de 2018, aceptado el 2 de diciembre de 2018

Cómo citar: Miller, J.C. (2018). Afecto y emoción en la ecología política urbana. Investigaciones Geográficas, 56, 80-99. https://doi.org/10.5354/0719-5370.2018.50835

\footnotetext{
Department of Geography and Environmental Sciences. Northumbria University. Ellison Place. Newcastle upon Tyne, UK. NE1 8ST. E-mail: jacob.miller@northumbria.ac.uk.

Este trabajo se realizó con el apoyo de Fulbright Commission (U.S. Department of State, U.S. Student Program); the Tinker Foundation, conjunto con The Center for Latin American Studies (University of Arizona); the Social and Behavioral Sciences Research Institute and the Institute of the Environment (University of Arizona)
} 


\section{Introducción}

El Mall Paseo Chiloé en Castro, el centro urbano del archipiélago de Chiloé en el sur de Chile, es un artefacto claramente vinculado con los flujos de capital neoliberal (De Simone, 2015). Mientras la sociedad de consumo produce varias consecuencias e impactos negativos para el medio ambiente (grandes cantidades de basura y residuos tóxicos, hasta el cambio climático), esos espacios urbanos también tienen una ecología propia. Según Jean Baudrillard (1988) los centros comerciales, mediante su infraestructura y ambiente controlado, generan "una primavera perpetua" (p. 34, traducción del autor). Más aún, el paisaje urbano es también una ecología política, en donde varios flujos ambientales se cruzan con las relaciones políticas de la vida contemporánea (Heynen, 2016). El crecimiento de una infraestructura de consumo corporativa en Chiloé, por ejemplo, depende mucho de la ecología política de la industria salmonera (Barton \& Fløysand, 2010) en donde una nueva cultura urbana de consumo crece al lado de los centros productivos (Hidalgo, Ther, Saavedra, \& Díaz, 2015). Los salmones industriales, en ese sentido, producen el sujeto del consumo del Mall Paseo Chiloé (Miller, 2018). Así ese artefacto urbano tiene unos lados ecológicos que merece atención en su conexión con otros flujos de inversión y comercio, retail corporativo particularmente, (Boddice, 2017, p. 13).

Sin embargo, este artículo pretende aproximar unos aspectos de esa ecología política urbana que no son siempre muy claros en la investigación: el rol de las emociones y el afecto. Como han señalado varios investigadores contemporáneos, el cuerpo es cada vez más un lugar central en la intersección de espacios urbanos y la política del sujeto en el contexto de capitalismo (Cena, 2018; Rendueles, 2017). Es decir, el cuerpo, con todo de lo que significa -particularmente sus lados emocionales y afectivos- es cada vez más central para la conceptualización de espacialidad y la producción de subjetividad política (Campos Medina, Silva Roquefort, \& Gaete Reyes, 2017; Lindón, 2009; Ginga \& Brizuela, 2017). Para la ecología política urbana, la tradición feminista señala el camino en como conceptualizar el cuerpo de esta manera (Elmhirst, 2011; Rocheleau, Thomas-Slayter, \& Wangari, 2013), y en años recientes ha habido más atención a los temas de emoción en las relaciones entre gente y sus ambientes, generando unas miradas hacia los lados íntimos en la vida cotidiana y su conexión a la ecología política (Harris, Martin, Polsky, Denhardt, \& Nehring, 2013; Smith, 2013). Al mismo tiempo, hay cada vez más enfoque en la ecología política urbana que toma en cuenta el cruce entre el mundo no-humano con los espacios políticos e infraestructuras urbanas (Heynen, 2016). Pero, hay pocos estudios todavía sobre la ecología política urbana que se enfoquen claramente en la emoción y afecto como elementos claves para entender la geografía de poder de hoy. Doshi (2017), por ejemplo, señala que es todavía poco estudiado (p. 125-126) y nos anima a explorar el gran potencial de investigación en esos campos.

Este artículo está de acuerdo con esas obras y pretende ser una guía para investigadores/ as que quieran estudiar esas dimensiones específicas. Aunque existen varias contribuciones teóricas sobre el afecto y emoción en las ciencias sociales (Bondi, Davidson, \& Smith, 2005; Campos Medina et al., 2017; Lindón, 2009; Pile, 2010), hay una escasez de obras que muestren claramente una metodología para su estudio. Jirón, Imilian, \& Iturra (2016) ofrecen un resumen breve de varios métodos que sí nos ayudan a pensar en los espacios cotidianos y las practicas que forman parte del paisaje corporeizado, pero el afecto y emoción no son conceptualizados exactamente. Más aun, hay pocas obras que pretenden guiar al investigador/a que esté interesado en aproximar el afecto y emoción y que pretenden anticipar los desafíos que se 
puedan presentar durante el trabajo del campo. Como ya hay varias contribuciones sobre las teorías del cuerpo afectivo y emocional, este artículo pretende ser tal guía. Tomando mi experiencia investigando el polémico Mall Paseo Chiloé y su contexto de cambio socio-espacial rápido, se ofrece algunos detalles en cómo se lleva a cabo tal estudio etnográfico que nos lleva hacia una ecología política urbana de emoción, afecto y los espacios del consumo.

\section{Materiales y métodos}

\section{Hacia una metodología crítica para el estudio de afecto y emoción}

En Poshegemonía, Beasley-Murray (2010) plantea la importancia del afecto (y emoción) en la política contemporánea latinoamericana (Pincheira Torres, 2013; Robertt, 2009). Las sensaciones son cada vez más importantes que la ideología, según Beasley-Murray (2010), y forman parte de una fuerza material que mantiene el paisaje de hoy. De manera interesante, especialmente en Chile, coincide con el desarrollo de nuevas tecnologías del consumo como los shopping malls (vea a páginas 20 y 188-189), que se despliegan junto con el desarrollo desigual en el contexto del neoliberalismo (De Simone, 2015). La política neoliberal se nutre de esos modelos de circulación e intervención en el paisaje. Así se ven vínculos entre filósofos como Gilles Deleuze y Michel Foucault, y los teóricos críticos latinoamericanos que han examinado los nuevos centros comerciales como Beatriz Sarlo $(1994,2009)$ y Tomás Moulian (1997, 1998). Ellos señalan la transformación en el contenido y función del poder hoy día, una propuesta que es clave para el estudio de centros comerciales y más allá, el mundo del consumo urbano.

Para el estudio del consumo, los centros comerciales y la ecología política urbana, es necesario entablar con esas nuevas teorías. Los estudios críticos clásicos del shopping mall son claramente basados en teorías representacionales, tal que el paisaje se puede leer como un texto que revela el poder social, político, económico y psicológico (Goss, 1993). Mientras los centros comerciales siempre serán lugares ideológicos, investigadores están cada vez más interesados en el dominio de sensaciones que emergen en el paisaje como un campo de intervención para el capital. Para el geógrafo inglés Nigel Thrift (2008), el poder capitalista contemporáneo funciona cada vez más a través de redes y tecnologías de afecto. La llamada Teoría No-Representacional se trata de las estrategias espaciales contemporáneas que también llaman la atención de Sarlo, Moulian y Beasley-Murray. El afecto puede quedarse en el consciente no-cognitivo y ser siempre elusivo (Thrift, 2008), lo han llamado atmósferas del background de la vida cotidiana (Anderson, 2014). Por eso es peligroso, qué mediante el diseño de paisajes urbanos, las sensaciones pueden impactar a los visitantes sin ellos saberlo. No se trata de la ideología o de la conciencia falsa, pero de cuerpos en movimiento adentro de atmósferas, diseños, eventos o prácticas, una situación donde el capital se realiza bio-política en la manera en que genera unos afectos entre ellos. Se trata de una "maquinación del afecto" (engineering of affect, en inglés) que mantienen ciertas formas de economía, política y vida social para Thrift (2008). Es un tipo de control social, pero en lugar de "conciencia falsa", está la bio-política que funciona cada vez más a través de sensaciones encarnadas (Luna Zamora \& Mantilla, 2017-2018).

Pero, para la investigación social, ¿cómo acercarse a algo que está definido como elusivo y no-cognitivo? Hay varias maneras de responder que tratan de revitalizar los métodos clásicos, hasta usar técnicos nuevos de investigar y conceptualizar el contenido. Por otro lado, otros han desarrollado una teoría de emociones. La emoción emerge cuando la atmosfera se cruza con el sujeto. Es decir, a veces sí es posible darse cuenta de la atmósfera afectiva y darle un nombre 
("me siento feliz, o triste" etc.). El afecto pasa y se transforma en emoción siempre, también, dando otra perspectiva en esos procesos. Además, los centros comerciales son también lugar para muchas otras prácticas espaciales-sociales, y la emoción puede aplastar la maquinación de afecto en ciertos momentos (Rose, Degen, \& Basdas, 2010). Aunque hay un debate académico sobre las diferencias del afecto y emoción, algunos han encontrado una manera de estudiar los espacios de consumo con ambos, que no son conceptos enemigos necesariamente (Wright, 2010).

En lugar de repasar esa discusión y debate teórico, el presente artículo presenta algunas estrategias para acercarse a esos dominios en términos de metodología. Se trata de una investigación realizada por el autor sobre el polémico Mall Paseo Chiloé en Castro, el capital urbano del archipiélago de Chiloé en el sur de Chile. Expondrá los métodos que fueron utilizados para acercarse a la vida de afecto y emoción en ambos lugares, métodos como la observación participativa; entrevistas y entrevistas móviles; y cómo usar materiales visuales. Primero, hay una descripción de las herramientas básicas que se pueden utilizar. Para entender mejor cómo funciona el mundo del consumo y su manifestación en la vida urbana cotidiana, yo quería experimentar con el pensamiento teórico mediante una pregunta de investigación amplia: ¿Qué rol juegan el afecto y la emoción para el funcionamiento de esos espacios?

\section{Herramientas}

La idea principal de la metodología de la investigación fue pasar tiempo alrededor y adentro de esos espacios para tener mi propia percepción y experiencia del espacio, pero también realizar varias actividades y entrevistas para tener una idea de que público existe allí, y empezar un experimento de aproximarse a la vida afectiva y emocional de esos espacios.
Encontré gente dispuesta a conversar conmigo y participar en la investigación (se realizaron 110 entrevistas en 2015).

Parte de la metodología fue realizar entrevistas grabadas y luego transcribirlas. Cuando fui a trabajar con intenciones de realizarlas, fui con un grabador de voz digital básico (vale $\$ 80$ US) que tenía a mano durante la entrevista. Es importante tenerlo a mano y listo, porque es posible que la gente que encuentras tendrán poco tiempo. Es para que el participante no cambie de idea de repente, o que no quiera esperar mientras usted busca el grabador enterrado en su mochila. Si Usted tiene éxito en conocer alguien que quiere participar, esté listo/a y preparado/a. Ande con dos grabadores, por si uno no sirve repentinamente. Ande con pilas extras también.

¿Qué hacer después de la entrevista? Ir directamente a copiar los archivos en otro lugar. Estos pueden generar una gran cantidad de datos digitales, entonces es importante tener una computadora, y un disco duro externo, que pueda guardar esa cantidad de datos. Sin estos, toda su data está en peligro. Yo trataba de copiar los archivos directamente y también trataba de transcribirlos justo después de la entrevista. Porque está recién llegado del momento mismo, y es una oportunidad de (1) empezar de analizar los materiales mientras los está generando, y (2) de conectar el transcripto con notas que sacaste sobre el espacio mismo.

Para escuchar y traducir las entrevistas, utilicé un software gratis, en donde se puede escuchar a los archivos a velocidad más lenta. Hay otras herramientas que pueden ser útiles para eso, como un pedal. Quizás se puede transcribir más rápido utilizando un pedal. Pero para mí, no es malo ir despacio y pensar más lento durante el proceso. No es solamente transcribir, pero pensar y empezar su análisis. "Lento no es malo". Haga un espacio de concentración, 
donde va pensando en cada palabra, cada sílaba y sonido, y la atmosfera afectiva y emocional de la entrevista.

Un cámara digital también se puede usar para documentar el espacio y, con algunas entrevistas móviles, necesarios para generar materiales. Esa también va a generar una cantidad grande de datos digitales, entonces es aún más importante tener la tecnología lista si va a usarla. Debajo se explica más en cómo usar la cámara.

Finalmente, utilicé unas herramientas análogas, como cuadernos para tomar notas $\mathrm{y}$ fotografías que formaron parte de una entrevista visual. Yo prefiero tres tipos de cuadernos, por lo menos: (1) Uno para sacar notas de la observación participativa y otras observaciones, (2) Otro cuaderno se reserva para poner algunos pensamientos de lo que significa todo, vinculado con teoría, (3) Un tercero es para anotar nombres y contactos de personas, y otros datos logísticos. Seguramente va a buscar una información o datos y sería útil dividir las notas en tal manera para facilitar eso. Puede guardar tiempo con ese detalle en cómo organizar su información.

\section{Observación participativa}

Observación participativa sigue siendo un método clave para entender la vida y política cotidiana. En los estudios recientes sobre la emoción en la ecología política, varios utilizan ese método junto con otros para generar resultados sobre esa relación que existe entre gente y sus ambientes (Dallman, Ngo, Paris, \& Thien, et al., 2013; Hayes-Conroy \& Hayes-Conroy, 2013; Nightingale, 2013). ¿Cómo se hace en contexto urbano? Ese método siempre ha sido orientado hacia sentir los ámbitos $\mathrm{y}$ atmosferas en donde habitamos. Para el estudio de los ambientes de la vida cotidiana y como se desarrollan desde una perspectiva del afecto, se puede a través de ese tipo de actividad, donde la idea es llegar a su propio entendimiento de tal actividad y espacio. Para algunos, ese método es suficiente para acercarse al mundo de afecto (McCormack, 2003; Wylie, 2005), pero ese camino metodológico corre el riesgo de estar ajeno a los temas de la subjetividad que también son fundamentales para la geografía humana y la teoría critica más allá (Mitchell \& Elwood, 2012). Más abajo hay una exposición de cómo resolver tal problema. Por ahora, este artículo se trata de algunas estrategias para la observación participativa que pretende afilar la vista sobre la vida afectiva en la vida cotidiana.

En mis estudios, he tenido la oportunidad de pasar largas cantidades de tiempo observando cómo se transforma y cómo se mantienen esos ambientes corporeizados afectivos. Hay dos tipos de aproximaciones. Primero: que Usted vaya donde sea. En ese plan, no hay un plan, o expectativa al 'qué hacer'. Solo anda y siéntese el espacio por sí solo, casi como la deriva de los situacionistas (Diaz, 2013-2014; Pyyry, 2018). Sea abierto a lo que pasa. ¿Pero cómo se documenta o capta esto como un proceso? Una idea es utilizar el grabador digital para hacer notas a sí mismo. Si hay algo que quiere anotar o describir, puede simplemente poner el grabador en lugar de sacar un cuaderno y lápiz, u otro aparato electrónico. Luego puede escucharlo y ponerlo en un cuaderno si desea. Andar con un cuaderno anotando cosas no es preferible, porque rompe con la experiencia en su proceso. Durante una visita de una o dos horas, por ejemplo, yo grabé cerca de cuarenta grabaciones-notas sobre el espacio y su ambiente, y lo hice sin romper tanto con la experiencia del mismo. Si está allí sacando notas todo el tiempo, no está participando realmente en/con el espacio. Se soluciona con el grabador digital.

Segundo, y, por otro lado, los cuadernos pueden ser útiles igual, pero en otro sentido. Como yo tenía varias semanas documentando los shopping malls $\mathrm{y}$ las actividades que se despliegan allí diariamente, también realicé varias 
estrategias para la observación. Como los centros comerciales son también lugares para no hacer nada, esto también es una forma de participación. Yo hice unas visitas a la misma hora y en el mismo lugar para entender que ritmos y actividades pueden pasar allí. Organicé el cuaderno, en tres secciones: una para la mañana y mediodía, una para la tarde y otra para la noche. A veces, hice micro-experimentos, donde fui a hacer observaciones en el mismo banco, por ejemplo, y generé una lista de eventos y materiales que conforman parte de ese espacio particular. De esa manera, después tendrá unos grupos de data en donde puede ver como los espacios cambian a través del día. Se puede crear un horario de visitación para ver como la vida afectiva del edificio cambia y se transforma.

Dentro de esas secciones en el cuaderno, también puede inventar unas categorías de experiencia que le interesen. Por ejemplo, usaba categorías que tratan el ambiente afectivo: sonido, texturas, eventos, colores, etc. De esa manera, va generando información sobre varios aspectos del espacio afectivo, y así ya está organizado según los conceptos que elige.

Hay la posibilidad de que esas dos aproximaciones se encuentren en conflicto. Por ejemplo, cuando el Mall Paseo Chiloé abrió sus puertas en 2015, yo intenté pautar un horario de visitación. Pero, la vida social del mall se concentró rápidamente, $\mathrm{y}$ siempre terminé conversando con la misma gente allí, haciendo imposible a veces a sacar notas durante ese tiempo. En lugar de evitar tales conexiones para atender al horario, hay que seguir lo que pasa cuando es necesario. Su horario puede ser flexible para tratar de mantener, lo mejor que pueda, una cobertura del tiempo y espacio. Trata de mantener el horario si tengas el tiempo; pero no sea sometido a ese orden, para seguir el fluyo de eventos afectivos. No pude, y no quise, evitar esas conversaciones, aunque fui con intención de sacar una serie de notas del ambiente.

\section{Entrevistas y entrevistas móviles}

Varios investigadores han utilizado entrevistas y entrevistas móviles para estudiar a la emoción y lo afectivo de la vida cotidiana. Para la ecología política urbana, algunos como Harris et al. (2013) han utilizado entrevistas móviles para acercarse al objeto de investigación: el césped de la casa privada en el paisaje suburbano. Visitar el espacio mientras conversan durante la entrevista añade aspectos emocionales en donde los participantes pueden describir como es su conexión con el espacio. Esos son resultados que no habrían aparecido sin la introducción del movimiento durante la entrevista. Se ven emociones mucho más complicadas que plantea Robbins (2007) sobre el mismo tema, en donde el sujeto del césped es conceptualizado en una manera mucho más estrecha. En todo caso, otros han avanzado estudios sobre el rol importante de la emoción para la ecología política mediante entrevistas fijas o tradicionales, en donde los investigadores hacen un esfuerzo para mantener un énfasis analítico sobre la emoción como parte clave de la relación entre humanos y sus ambientes (Dallman et al., 2013; Hayes-Conroy \& Hayes-Conroy, 2013; Nightingale, 2013).

Un problema para el estudio de geografías del afecto es como acercarse a algo que está definido por ser fluido y elusivo. Mientras entrevistas también pueden ser leídas por su contenido o tono emocional, también lo afectivo genera un marco. Cuando los participantes no pueden encontrar la palabra correcta para explicar algo, o tienen dificultades, allí se puede pensar en la fuerza afectivo que, quizás, no puede ser siempre nombrado. Woodward, Jones, \& Marston (2012) leen eso en las entrevistas de Sitrin (2006) sobre el movimiento de los trabajadores argentinos y las fabricas recuperadas después de la crisis de 20012002. Cuando en la entrevista, se pronuncia que "Look, I can't explain it to you" (Woodward et al., 2012, p. 216), significa la presencia de algo más, de un sitio de afectos 
que abruma el sujeto. En esa manera, es posible re-pensar la entrevista para ver esos momentos afectivos claves, además del contenido que se trata explícitamente sobre las emociones.

En las entrevistas, también puede provocar reflexiones sobre la vida afectiva y emocional al centro comercial. Mientras la entrevista puede ser rica si empieza de escucharlo para los contenidos afectivos y emocionales, la entrevista móvil es una manera de generar otro tipo de información con los participantes de investigación. Anderson (2004) explica como ese movimiento genera otras oportunidades para relacionarse el investigador y el participante. En su go-along, Kusenbach (2003) realiza unos viajes a dentro de la vida cotidiana de los participantes para entender mejor lo de siempre de un barrio, viendo varios cualidades y materiales en el proceso. Rose et. al., (2010) adaptan la idea para un estudio enfocado sobre los afectos y emociones de unos centros comerciales en Inglaterra. Visitando los centros comerciales creaba un espacio para ver y sentir lo que pasa allí (cómo se mueven y qué hacen los participantes). Se conversan mientras recorren el espacio, con la agenda dirigido por el participante. Llamándolo un walkalong, van caminando juntos en lugar de hacer una entrevista fija y sedentaria, cara a cara. Se ve lo que se hace en el espacio, también en lugar de solo escuchar lo que se dicen que se hacen allí.

Rose et al. (2010) también utilizaban cámaras para que los participantes saquen fotos del espacio, que luego serán útiles como aportes en una entrevista subsecuente. En un estudio sobre un centro comercial en Buenos Aires (el Abasto), yo le di mi cámara a los participantes y como Rose et al. (2010) les pide que saca fotos de cualquier cosa que le llama más la atención (Miller, 2015). Luego, la idea es usar las fotos durante una entrevista después, para que ellos pueden explicar su fotografía, algo que se genera aún más datos sobre su relación al lugar. Puede ser difícil obtener ese tipo de entrevista. Obviamente, es bastante invasivo al espacio y tiempo del participante, más aún si pretende realizar la entrevista subsecuente. Muchas veces, yo fui a tratar de realizar una entrevista de ese tipo. Así, esperaba fuera del edificio intentando conversar con gente que iba en dirección de las entradas. Después de algunas preguntas iniciales de introducción, propuse la entrevista móvil durante su visita. Es más difícil, porque va a hablar a solo la mitad de la gente allí. Seguramente no todos van a querer o tienen el tiempo para participar en el estudio, una entrevista corta o algo más largo como una entrevista móvil. Pero vale la pena cuando alguien le gusta la idea y participa en esa manera. Por uno, extiende la experiencia de investigación, dando más oportunidades para que pueden elaborar sobre el espacio. Además, hay la posibilidad de que ellos pueden ver algo y lo señalan mientras explican el significado. Así aparezcan cosas que no hubieran sido vistos o entendidos sin ese experimento.

Algunas veces los participantes se olvidaron de la cámara completamente. Quizás hay que recordarles de la cámara como algunos. Cuando saquen fotos, es importante organizarlas pronto después, para que no se mezclan esas fotos con las que Usted también haya sacado. Yo también mantenía el grabador de voz encendido durante esa actividad, un texto que va a captar muchos otros sonidos e interrupciones que también serán útiles para entender las atmósferas del mall. Hay un archivo que va más allá de solo las voces y sus tonos durante la entrevista. Esos sonidos también con voces de otras fuerzas que componen la atmósfera afectiva del paisaje comercial. Genera un archivo de los materiales afectivos que forman parte de la atmósfera. Varias veces mientras la transcripción he encontrado la entrevista rota por alguna intervención del espacio mismo. En la vereda, pasan los autos y camiones grandes, con sus motores ruidosos. O el sonido de los juegos en el Mall Paseo Chiloé. 


\section{Entrevista con fotos}

Para acercarse al mundo emocional adentro de una ecológica política, Graybill (2013) utiliza la producción de textos como una manera de hacer mapeo cognitivo. Pide que los participantes hagan mapas, dibujos y notas para representar las conexiones entre ellos y el paisaje. Aparte de las fotos de la entrevista móvil mencionadas arriba, hay otras maneras de utilizar materiales visuales para provocar la emoción y el afecto en una entrevista (Harper, 2002). En el estudio del Mall Paseo Chiloé, yo utilizaba fotos en la metodología de "Q-sort" (Robbins \& Krueger, 2000), donde participantes tienen que ordenar varias frases o imágenes según un apunte. Por ejemplo, me interesaba como los participantes se sienten con respeto al proceso de transformaciones en el paisaje de Chiloé, desde un campo agrario hasta los nuevos espacios urbanos, industriales y de retail. Entonces les pedía que miran una mezcla de fotos de varios tipos, fotos como ejemplos de varios ritmos o corrientes que forman parte del Chiloé contemporáneo, con fotos del campo; fotos de espacios urbanos; actividades turísticas o de patrimonio. Tuvieron que poner en orden según esa frase: "Prefiero que Chiloé sea así".

En la metodología de Q-sort, la idea es que eligen una foto con calificación máxima $(+3$ en ese caso) por un lado, y mínima (-3) por otro lado. Luego, dos fotos que también son positivas, pero no lo máximo $(+2)$; tres fotos con calificación $(+1)$; cuatro fotos neutras (0); y lo mismo hacia el lado negativo, donde colocan las fotos que no le gustarían en el paisaje de Chiloé, terminando en una pirámide o diamante (figura 1). Pueden explicar sus decisiones mientras lo hacen, o después. Para notar como lo hicieron, yo saqué una foto de su pirámide y luego anoté en una tabla, donde cada foto tiene su número.

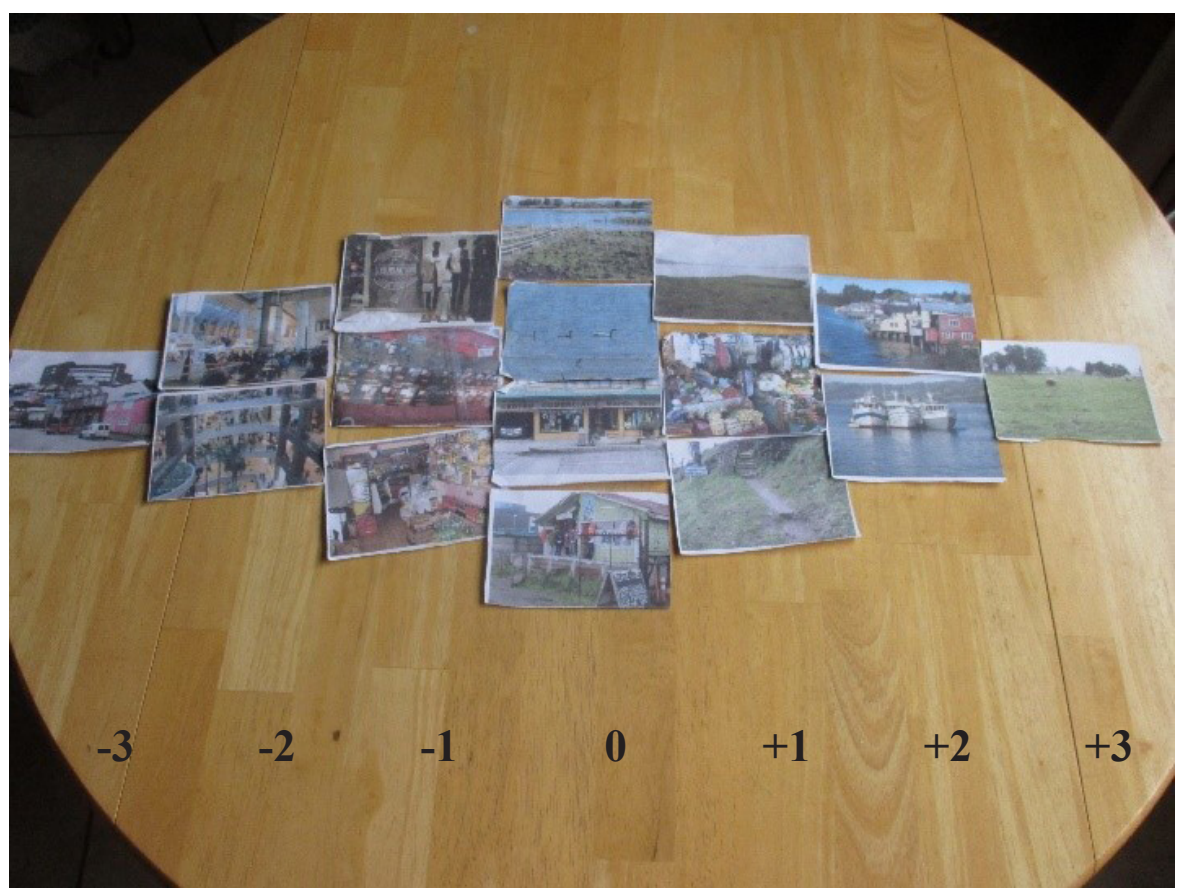

Figura 1. Diamante de fotos ordenados por un participante de entrevista según la frase "Prefiero que Chiloé sea así". Fuente: Elaboración propia, 2018.

Figure 1. Photo diamond ordered by interview participant following the prompt "I prefer Chiloé to be like this". Source: Own elaboration, 2018. 
Si Usted va a querer realizar análisis estadísticos, es obviamente importante que los participantes siguen esas reglas estrictamente. Quizás no le suene complicado. Pero yo encontré unos desafíos, desafíos que también forman rutas a otros resultados. Algunos no querrían obedecer las reglas y preferirían su propia forma en ordenar las fotos. Al principio, intenté de pedir que lo piensan en forma estricta, pero me di cuenta que no es prudente. Fue une perdida de oportunidad para conversar, en lugar de repetir las reglas otra vez y otra vez. Un participante negó la idea totalmente, pero la entrevista fue muy rica y útil igual. El punto es ser flexible con los participantes si no tiene intenciones de realizar análisis en que se necesita mantener reglas duras.

Otra sugerencia es explicar su pirámide con su propia voz durante la conversación/ entrevista. Es porque cuando Usted escucha el audio después, va a escuchar un participante hablando de una foto, por ejemplo, pero no siempre será claro que foto exactamente. Si usted, durante la entrevista, simplemente comenta lo que están hablando, es más fácil cuando tener la certeza cuando lo escuche luego. El desafío es no hablar demasiado, porque la idea, obviamente, es que los participantes pueden elaborar el significado de las fotos y como se sienten en relación a ellas. Entonces, se puede intentar a intervenir en manera muy mínima, pero le ayudarían luego. Vea por ejemplo el siguiente fragmento de una entrevista:

Carmen: Que lindo el paisaje ese. [2:00:42].

Autor: ¿Con la oveja?

Carmen: Sí. Que lindo.

Autor: Le gusta esta foto del campo.

Carmen: ¡Me gusta todo lo que es campo! (31 de julio 2015, Biblioteca de Castro)
Luego, se habla de la diferencia entre el campo de su memoria y la vida contemporánea de Castro, que ha crecido rápidamente en las últimas décadas. Había varias fotos del campo que podría haber sido, pero aquí se ve la intervención mínima que queda claro cuál foto exactamente fue lo que le llamó la atención primero; la foto de una casa rural con una ovejita sentada en el pasto.

Finalmente, hay que pensar en cómo y cuándo realizar ese experimento. Primero, es necesario tener espacio para exponer las fotos, sobre una mesa y banco donde sentarse. Puede ser difícil si están afuera, donde el viento o la lluvia también pueden intervenir. Una idea es realizar esa actividad después de una entrevista, para ver que más apareciera que no hubiera sin las fotos. De esa manera, se puede pensar en cómo su actitud sobre el mall está vinculado a otros procesos de transformación del paisaje. Pero, por otro lado, puede ser útil también empezar una entrevista así, donde las fotos guíen toda la conversación. Ambos estilos pueden ser útiles. Una cosa es saber cuánto tiempo Usted va a tener con los participantes. Si es poco tiempo, quizás es mejor usar las fotos al principio, y con participantes que tengan más tiempo, puede esperar al final.

\section{Resultados}

Ahora se exponen algunos resultados que corresponde a cada método presentado arriba: observación participativa, entrevistas, entrevistas móviles y entrevistas con fotos. Aunque el propósito de ese artículo no es exponer los resultados totales del estudio (Miller 2018, 20182019), así se ve los datos diferentes que se generan tales métodos con respeto al estado afectivo y emocional de los participantes, el investigador y los espacios del edificio mismo. 


\section{Observación participante}

I hear the squeaking at M's Café, squeaking from the toy rides.

A kind of humm-buzz comes from the mall, can't really hear the music that much.

Now there are padded seats in the 3rd [floor] patio around the pillars. [Oct, 17, 2015, Mall Paseo Chiloé. Fuente: notas de terreno].

New ride in 1st floor has loud music when coin goes in. I hear it from the door. [November 9, 2015, Mall Paseo Chiloé. Fuente: notas de terreno]

En la observación del espacio del Mall Paseo Chiloé, se ve algunos elementos que se puede caracterizar como la maquinación del afecto mediante el diseño y arquitectura. Las escaleras mecánicas, por ejemplo, y las ventanas grandes que dan vista al mar, son mecanismos que ofrecen algo más a los visitantes. Sin embargo, esos resultados arriba, sacados del cuaderno de notas de campo, muestra algo diferente. A pesar de toda la manipulación del espacio por capital, hay unos mundos autónomos adentro (Massumi, 2002), que hay mundos de afectos y erupciones que también componen el ambiente. En Miller (2014), postulé en situaciones parecidas en el caso de estudio sobre el Abasto Shopping mall en
Buenos Aires, Argentina, la posibilidad que ese mundo afectivo y emocional a veces vence a los modelos de afecto maquinado (Rose et al., 2010). Siguiendo Deleuze \& Guattari (2006/1980), postulé que ese tipo de carácter es algo emergente del espacio mismo. Con respeto al arriba, se ve eventos no esperados que son siempre un desafío a los planes de los empresarios y políticos que están interesados e invertido en el paisaje de consumo. Por ejemplo, hay unas tecnologías para entretener a los niños pequeños, el juego en figura 2 , que parece como un vehículo que se mueve y se activa con unos pesos, muchas veces con una canción que toca durante el viaje y otros elementos como luces y cosas materiales para tocar. Mientras siendo algo para llenar el mall como lugar de experiencias -en ese caso, para la familia- algunas de esas máquinas también necesitaban grasa para su mecánica interna. Esto provocó unos sonidos duros, repetitivos y de tono muy agudo, sonidos no agradables. Eso obviamente es una interrupción al esquema urbano comercial. Mientras esos son dirigidos para promover un ambiente amigable (algo para entretener los niños), sin embargo, aparece, de repente, un desafío que requiere un tipo de trabajo para controlar. El punto clave es que los afectos son a veces excesivos de los deseos del capital de retail. El poder de ese no es tan fuerte como se imaginan algunas teorías críticas del consumo (Miller, 2014).

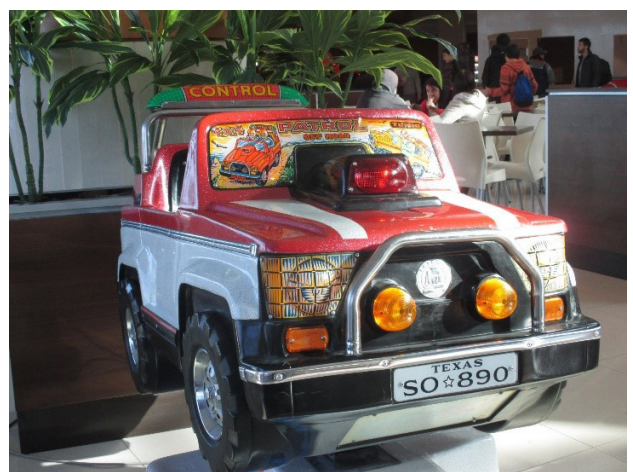

Figura 2. Juego para niños: necesita grasa; Mall Paseo Chiloé. Fuente: Elaboración propia, 2015.

Figure 2. Kid's ride: needs grease; Mall Paseo Chiloé. Source: Own elaboration, 2015. 


\section{Entrevistas}

Llegué a Castro en marzo de 2015, antes del abrimiento del mall más tarde en ese año. En los primeros meses conocí gente en otros espacios para solicitar conversaciones y entrevistas sobre el mall (como la plaza o en la calle) y cuando se abrió también se realizaba entrevistas adentro con participantes. Con las entrevistas, yo eventualmente empecé de generar algunos temas que me parecía frecuente: (1) el mall como inevitable, (2) el mall como un avance, (3) el rol de la movilidad entre Chiloé y el continente, o el tema del "aislamiento" de Chiloé, y (4) la figura del ciborg, un concepto de Haraway (1991) que adapto aquí como categoría interpretado para pensar en un futuro sujeto Chilote que no es simplemente engañado por el sistema capitalista, pero que se mantiene viva en muchas maneras al lado and con el paisaje consumista nueva. Una vez que llegué a esos temas, fui a repasar las entrevistas otra vez para ver las conexiones que existen entre los participantes y la frecuencia de cada tema a través de todas las entrevistas.
Mientras estaba codificando las entrevistas, estaba también pensando en otro ejercicio analítico: generar unas estimaciones del estado de ánimo con respeto al mall. La idea fue leer y entender los datos con un enfoque sensacional, buscando lo afectivo y escuchando para la emoción. Leyendo los textos así - haciendo unas observaciones de su contenido, discurso y tono - traté de estimar su estado de ánimo, si fuera más opositor o partidario, o si fuera más ambivalente sobre el tema. Mi interpretación termina con cinco grupos en donde cayeron esos participantes que conocí al azar adentro del mall o en otros espacios en Castro en 2015 ( $n=72)$. Un grupo es formado por los opositores fuertes al mall, que tenía críticas muy duras. Otro grupo son los partidarios, los que apoyan al mall fuertemente y no tienen nada negativo para decir. Sin embargo, un gran parte de los participantes fueron más ambivalente; hay algunos que estaba ambivalente en manera completa. Hay dos grupos más que también son ambivalente, pero uno es un poco más opositor y el otro un poco más partidario. Segmentada por grupos de edad también, se ve la distribución en tabla 1.

\section{Tabla 1}

Estimación de Estado de Ánimo con respeto al Mall Paseo Chiloé con participantes al azar en Castro en 2015; no incluye los profesionales o líderes cívicos quien también participaron. Fuente: Elaboración propia, 2018.

Table 1

Feeling Estimate with respect to the Mall Paseo Chiloé with participants chosen randomly in Castro in 2015; does not include professionals or civic leaders who also participated in interviews. Source: Own elaboration, 2018.

\begin{tabular}{lllllll}
\hline $\begin{array}{l}\text { Grupo } \\
\text { de Edad }\end{array}$ & Opositor fuerte & $\begin{array}{l}\text { Ambivalente } \\
\text { pero un poco } \\
\text { más opositor }\end{array}$ & $\begin{array}{l}\text { Ambivalente } \\
\text { pero un poco } \\
\text { más partidario }\end{array}$ & $\begin{array}{l}\text { Partidario } \\
\text { fuerte }\end{array}$ & Total \\
\hline $18-25$ & 2 & 3 & 1 & 2 & 0 & 8 \\
$26-35$ & 1 & 3 & 3 & 5 & 3 & 15 \\
$36-45$ & 2 & 2 & 1 & 10 & 11 & 26 \\
$46-55$ & 0 & 1 & 4 & 3 & 4 & 12 \\
$56+$ & 0 & 0 & 0 & 4 & 7 & 11 \\
Total & 5 & 9 & 9 & 24 & 25 & $\mathrm{n}=72$ \\
\hline
\end{tabular}


Se puede observar que la mayor parte de los participantes fueron más partidarios al mall. Con esa metodología, aprendí algunas razones porque, que muchas veces fueron cuentos íntimos sobre la vida cotidiana en Chiloé. El tema de aislamiento fue muchas veces comunicado con referencia al viaje a Puerto Montt, donde los viajeros aprovechan de comprar cosas en los malls y las tiendas grandes como Falabella y Ripley que antes no había en Chiloé. Me han contado varios que el viaje no es muy cómodo, dura todo el día y puede ser caro pagar los pasajes para una familia entera. Además, algunos se quejaban de los precios más altos en la isla debido a esa geografía. Hay una percepción que el resto del país tiene acceso una infraestructura mejor, $\mathrm{y}$ Chiloé merece su propio mall. Ese discurso lo llamaría el derecho para consumir, y es lo que explica muchos de los partidarios del mall según mi interpretación.

Por otro parte, fui a buscar los que estaba involucrado en la polémica en los últimos años, incluyendo miembros del Colegio de Arquitectos, grupos ciudadanos y otros profesionales y líderes cívicos. Dependiendo en quien, yo les entrevisté a ellos para entender mejor varios aspectos de la polémica, incluyendo los detalles del edificio mismo y la historia muy larga y confusa de su desarrollo. Con algunos, yo también utilicé la fotografía durante la entrevista (hay más abajo). Creo que hay la posibilidad aproximar los espacios emocionales y afectivos en esas entrevista cortas con el público al azar y también con los profesionales. El mall seguramente golpeó un nervio, en lo que es la conexión que distintas comunidades tienen con el paisaje mismo y su significado para el desarrollo sostenible y justo. Muchos de los ambivalentes y opositores lamentaban los procesos de cambio que pasa en Chiloé hoy día, en donde una nueva cultura urbana reemplaza lo rural que siempre fue el fundamento para la identidad Chilota. El Mall Paseo Chiloé, además de ser ilegal según algunos, el Mall es también injusto por su impacto negativo en el paisaje patrimonial. Fue muchos tonos de ira, desconfianza y chasco para esos participantes que se opusieron al mall y tenía mucha razón para hacerlo, pero no fueron exitosos en pararlo. El supermercado Líder en el subsuelo del Mall se abrió en abril 2015 y la primera fase del mall mismo en Agosto de ese año. En noviembre, la Corte Suprema de Chile emitió el juicio en favor de Pasmar, la inversionista, y los permisos que le dieron la Municipalidad de Castro que fueron cuestionados por el Colegio de Arquitectos y otros grupos cívicos.

\section{Entrevista móvil}

En una entrevista móvil, se veía cosas banales que aparecieran que quizás nunca hubiera encontrado en una entrevista sedentaria. Ofrezco dos ejemplos. Primero, yo fui al Supermercado Líder en el subsuelo del Mall con Karina ${ }^{1}$ (4 de mayo 2015). Ella es de Castro y yo aprendería más tarde que se opone al Mall, pero ese día se veía visitando para buscar los pañuelos para sus niñas de dos años. Aunque fue una visita corta y aparentemente sin ocasión, a la caja ella recitó su número de miembro del club que tiene Líder para captar datos sobre el consumidor. Aunque fue su primera visita a ese mercado particular, ella ya estaba marcado por los ritmos de la tienda y su tecnología. Muchos participantes, y también en conversaciones, ya están acostumbrado al sistema de consumo que encontraron en Puerto Montt, o en otras ciudades, y que quieren traer a la isla. El otro caso también es relacionado con el tema de género en el mall. Una tarde, conocí a Rosa llegando al mall (el 16 de agosto 2015). Necesitaba algo para su casa, me dijo, que iba a Falabella. Mientras ella me dijo que había un solo producto para buscar, terminamos vagando

1 Los nombres de los participantes al azar fueron cambiados para mantener el anonimato de ellos, mientras muchos profesionales y líderes cívicos quisieron que se utilizara su nombre real. 
por la tienda, en los otros departamentos y de repente mirando a productos electrónicos (televisión, computadoras, cámaras). Finalmente, pasamos por la ropa para hombre, donde paramos para mirar unos pantalones. "Compro para mi esposo" me dijo mientras tocaba el material y pensaba en el precio. Salimos de la tienda sin comprar nada, pero habiendo explorando varios ambientes de la mega-tienda. En ambos casos, se ve la importancia del género en formar sujetos del mall, en lo que buscan allí y porqué. Eso es importante de pensar en cómo la tecnología de manipulación de afecto y emoción no es siempre universal (Tolia-Kelly, 2006), pero se cruce con otros identidades y políticas que también forman parte del mall y de la ciudad más allá.

\section{Entrevista con fotos}

Finalmente, aquí se expone algo de la entrevista con fotos. Con Carmen Antiñanco, Presidenta de su Junta de Vecinos en Castro y conocida militante por muchos años, conversamos más de una hora en la Biblioteca de Castro antes de que miramos a las fotos. Cuando eligió la foto con las ovejas, fue solo el inicio de una serie de recuerdos que provocó. Luego conversamos sobre la foto de los palafitos de Gamboa (figura 3), y de repente me dice con ánimo, "¡viví en uno!... antes del terremoto en 1960". Explica que a lo largo de su vida se recuerda de esos años y como se relaciona con el medio ambiente en otros lugares en Chiloé. Conversamos por mucho tiempo sobre la vida en Chiloé y el polémico Mall Paseo Chiloé. Cuando volvimos a la foto de los palafitos, que ahora son transformados algunos en hoteles boutiques y restaurante gourmets que corresponden con un auge en turismo, Carmen añade esos reflejos:

"Por la vista que tiene! Imagínese de la gente de acá arriba, de aquí está sentada en la terraza, y mirando al mar, y mirando a la gente que está mariscando más allá. Es muy bonito poh', respirando aire puro. [entonces, en tono más sombrío:] Así los Chilotes no valoramos nada lo que tenemos. Tiene que venir otras personas a valorar" (31 de julio 2015, Biblioteca de Castro).

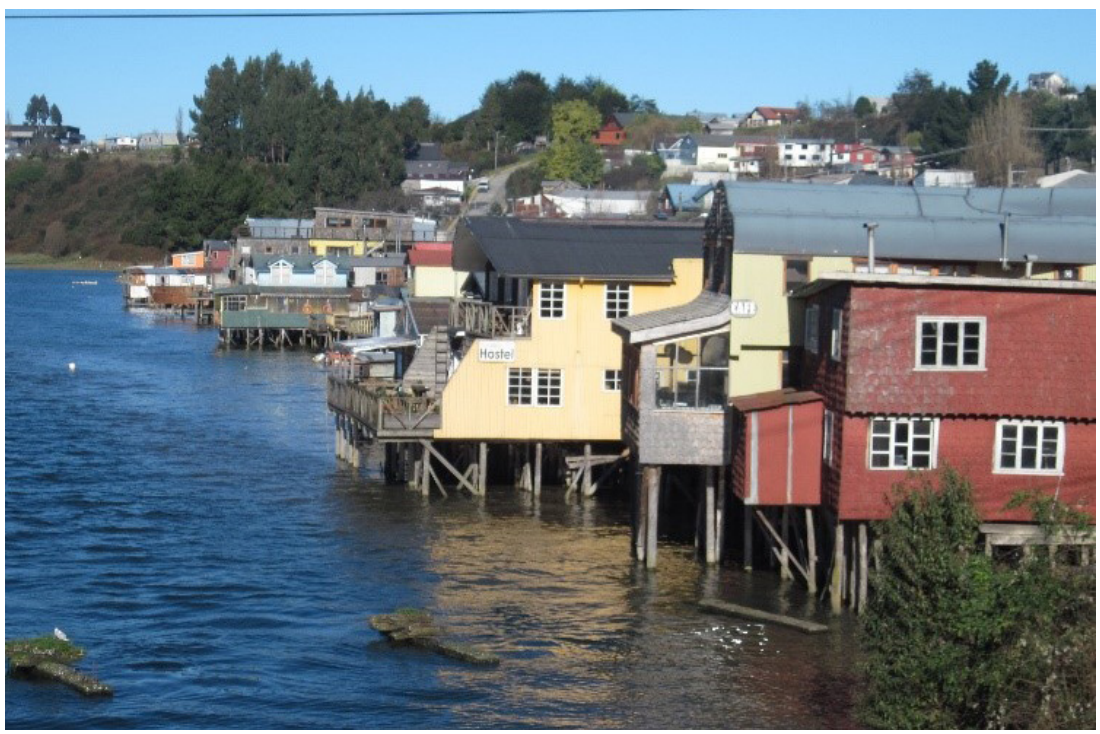

Figura 3. Palafitos de Gamboa, Castro. Fuente: Elaboración propia, 2013.

Figure 3. Gamboa Palafitos, Castro. Source: Own elaboration, 2013 
Entonces, esa reflexión en el paisaje da una idea de cómo esta pensando ella en términos alrededor de la relación que existe entre gente y el paisaje. Mirando las fotos con ella muestra algo que le afecta, una sensación de melancolía, desacuerdo o chasco, algo que explica como se ha llegado a un edificio tan absurdo como el Mall Paseo Chiloé. Vale decir que la foto del Mall Paseo Chiloé imponente con respeto a la ciudad fue uno de los más negativos para ella (-2), detrás solo la foto del interior del Costanera Center en Santiago (-3), una foto de un interior cualquier de los malls de hoy. Hay algo de nostalgia en esas palabras, una emoción que aparece en otra política de emoción en la región (Colin, 2017).

\section{Discusión}

En otro artículo (Miller, 2018) examino la ecología política de la industria salmonera en Chiloé y la emergencia de un paisaje urbano cada vez más orientado a consumo, y que parece cada vez más parecido a otras ciudades chilenas. Los salmones industriales tienen un efecto amplio por la manera en que se unen sistemas distintos: geografía física, la infraestructura de la industria salmonera en el contexto de neoliberalismo, y nuevos practicas espaciales vinculados con el uso de efectivo, crédito, mercancías y retail corporativo. Los salmones no solo se unen esos materiales con mercados distintos transnacionales también, pero producen la posibilidad de algo emergente en términos de nuevos sujetos (socio-culturales, políticos y económicos) que no habrían aparecido sin esa producción de conjunto ("assemblage", según Bruno Latour). Pero, ese enfoque no incluye tanto enfoque al tema del cuerpo y el mundo emocional y afectivo asociado con esa nueva ecología política urbana. El articulo presente empieza el trabajo de enfocarse aquí con más claridad y enfoque metodológico para completar tal perspectiva.

También, en otros artículos yo y otros investigadores/as hemos desarrollado resultados sobre el espacio nuevo que emerge en investigaciones que utiliza esos métodos etnográficos (Miller, 2014, 2015; Rose et al., 2010). Crea la oportunidad de que información nueva puede surgir qué no habría aparecido sin esos métodos. Además, esos métodos son adecuados para el estudio de emoción y afecto en la ciudad de hoy. Con esa metodología, creo que fue posible para mí comprender varias perspectivas sobre la polémica Mall Paseo Chiloé. Yo aprendí que los partidarios del Mall tienen algunas razones legítimas que explican, en parte, sus deseos, y que tienen que ver con la relación entre el archipiélago y el continente y la política de desarrollo que todavía utiliza el concepto de avance para promover tales mega-proyectos. Sus deseos tienen que ver con la conectividad y flujo de bienes, y con lo que ahora algunos se llaman el aislamiento relativo con los centros urbanos con infraestructura técnicos. Corre un discurso en ese grupo de los partidarios del mall que se puede describir como el derecho para consumir. No es solamente un discurso, pero está vinculado a varias experiencias en el paisaje urbano. El viaje a Puerto Montt, según ellos, no es simplemente incómodo o caro para algunos, pero una injusticia (Miller, 2018).

Mientras había una mayor cantidad de gente partidario al mall, la gran mayoría fueron parte los tres grupos ambivalentes. El Mall Paseo Chiloé como mal necesario fue un estribillo a través de las entrevistas. Quiero también señalar que la mayoría de los profesionales o lideres cívicos que busqué que participaron estaban en contra del Mall, pero no aparecen en la tabla 1 porque eso incluye solo los participantes que conocí al azar en los espacios urbanos de Castro. Con Carmen, aprendí detalles íntimos de su perspectiva sobre la transformación de la ciudad. Mirando las fotos juntos, se veía una sensación de chasco y tristeza al fondo. Los procesos urbanos, se puede decir, están creando un sujeto que para ella "no valora lo que tenemos" según ella, que fue algo frecuente que dicen los opositores 
al mall. Son frustrados, y lamentan los cambios recientes que ahora se hace una sociedad cada vez mas urbano y parecida a otras ciudades de Chile. Hay una sensación de perdida adentro de los procesos de transformación, y eso les empuja a tomar acción en la producción de espacio.

En todo caso, la figura del Ciborg me parece útil, porque no quiero considerar los partidarios del mall como simples engañados por el capital de retail. Adentro de los partidarios del mall, hay una conciencia de que hay algo para defender. Es decir, ellos no teman el Mall Paseo Chiloé, pero dicen que la cultura depende en ellos mismos, que un solo edificio no puede cambiar su identidad. Quizás es una subestimación del poder de la arquitectura. El Colegio de Arquitectos, por ejemplo, fueron opositores al mall por su visión de una practica ética de arquitectura, en cambio del corriente de libre comercio que domina la producción de espacio en Chile neoliberal de hoy. Mientras todo eso desdobla en la cultura, hay una industria turista que vende Chiloé como lugar todavía prístino, único y misterioso, creando otro circuito de consumo de gente que valora a Chiloé con un lugar único. Espero que los partidarios optimistas -los sujetos ciborgpueden florecer, pero también temo que el capitalismo contemporáneo crece su tecnología a través del conocimiento de esos campos de vida al mismo tiempo.

En conclusión, es necesario también preguntar sobre la nueva ecología política urbana que incluye los materiales, objetos y atmósferas encarnadas de la infraestructura de consumo corporativo. Es decir, ¿En qué sentido es el paisaje afectivo, lleno de objetos y no-humanos, también una ecología política en si misma? Para Bennett (2010), una "ecología política de cosas" (political ecology of things, en inglés) se dedica a ese mundo "más-que-representacional" (Lorimer, 2005) que siempre presenta un desafío a las estrategias y tecnologías espaciales humanas. Esa investigación del Mall Paseo Chiloé trata de incluir ese mundo junto con los mundos emocionales y las percepciones que tienen los habitantes sobre su propio paisaje y las ecologías que rodea y forma Chiloé. El crecimiento de las ciudades y espacios urbanos es vinculado con una disminución de vida rural y comunitario, mientras el impacto es desigual a lo largo del archipiélago (Daughters, 2015, 2016; Hidalgo et al., 2015). Para algunos, el Mall se impone con un discurso bastante claro de "modernidad", que presenta un optimismo. Para los críticos, es un optimismo mal otorgado, que falta una conciencia de la importancia del paisaje arquitectónica y patrimonial. En todo caso, el mall ofrece una nueva ecología política: una de bienes importados y retail corporativo que incluye ambientes interiores nuevos con escaleras mecánicas, tarjetas plásticas de crédito, junto con una infraestructura tecnológica de finanzas y deudas. Es todavía para ver lo que van a hacer los Chilotes con esa infraestructura nueva, y que relaciones van a aparecer alrededor de su ambiente seductor y corporativo.

\section{Conclusiones}

El propósito de ese artículo no es repasar la literatura grande en las ciencias sociales, ni exponer los resultados exhaustivos (Miller, 2018, Miller, 2018-2019). En cambio, la idea fue ofrecer una guía para los investigadores que quieren saber cómo se realice una investigación en tal manera. En todo caso, hay algunas conclusiones que se puede sacar de los resultados que si aparecen aquí. Lo importante es darse cuenta que el mall, como tecnología espacial dedicado al capital y los flujos de la gobernanza neoliberal, es siempre un proyecto en/de proceso (Palmett Plata, 2015), que no tiene nada realmente "mágico" (Goss, 1993) que asegura el poder capitalista neoliberal. Es decir, la "magia" que existe en el mundo consumista es un producto sin garantía; eso se ve cuando el mall se cae a pedazos en la práctica cotidiana debido a varias irrupciones no esperadas (Miller, 2014). En ese sentido, la teoría del afecto merece más 
atención como una manera de expandir nuestro concepto de espacio y su rol ontológico en la política contemporánea de cuerpos y el sujeto urbano (Aubán-Borrell, 2017; Huiliñir-Curío \& Zunino, 2017). Adentro del edificio, donde hay un nivel de control máximo, se ve esa realidad de la "rizoma" de Deleuze y Guattari (Diaz 2013-2014). Aquí se ve una ecología política parecida a Bennett (2010), en el sentido que el sujeto del consumo se ve como un producto de varios flujos y estados afectivos entre cuerpos y ambientes. Igual que los participantes son muchas veces ambivalentes con respeto a esa megainfraestructura. Al mismo tiempo, ese estudio muestra la posibilidad de utilizar esos métodos más asociados con la vida emocional. Muchas veces la polémica alrededor del mall depende en prácticas espaciales, que incluyen la conectividad entre el archipiélago y el continente, y también las prácticas como el turismo y alrededor de Patrimonio. Más que eso, esa metodología también se muestra lados importantes sobre otros temas socioespaciales, sobre el género, por ejemplo. Es decir, hay varios sujetos del mall y no uno sólo. Se necesita una metodología que puede atender a esa multiplicidad para tener una perspectiva más amplia de su importancia y sus consecuencias adentro de la vida urbana de hoy. Parece que la ecología política urbana podría utilizar fácilmente ese tipo de metodología para comprender mejor la emoción y afectivo.

\section{Agradecimientos}

El autor agradece a las siguientes instituciones que apoyaron con fondos la presente investigación: Fulbright Commission (U.S. Department of State, U.S. Student Program); the Tinker Foundation, en conjunto con The Center for Latin American Studies (University of Arizona); the Social and Behavioral Sciences Research Institute and the Institute of the Environment (University of Arizona).

\section{Referencias}

Anderson, B. (2014). Encountering affect. Capacities, apparatuses, conditions. Farnham and Burlington: Ashgate. ISBN: 978-0-7546-7024-7

Anderson, J. (2004). Talking whilst walking: a geographical archaeology of knowledge. Area, 36 (3), 254-261. https:// doi.org/10.1111/j.0004-0894.2004.00222.x

Aubán-Borrell, M. (2017). La dignidad de los márgenes. Aproximaciones afectivas a la ciudad informal. Revista invi, 32 (9), 67-89. https://doi.org/10.4067/s071883582017000300067

Barton, J.R. \& Fløysand, A. (2010). The political ecology of Chilean salmon aquaculture, 1982-2010: A trajectory from economic development to global sustainability. Global environmental change, 20(4), 739-752. https://doi.org/10.1016/j. gloenvcha.2010.04.001

Baudrillard, J. (1988). Jean Baudrillard. Selected writings. M. Poster (Ed.). Stanford CA: Standford University press. ISBN: 0-8047-4273-1

Beasley-Murray, J. (2010). Poshegemonía. Teoría política y América Latina. Buenos Aires, Barcelona, México: Paidós. ISBN: 9501289117, 9789501289114

Bennett, J. (2010). Vibrant matter: A political ecology of things. Durham and London: Duke University press. ISBN: 978-0-8223-4633-3

Boddice, R. (2017). The history of emotions: Past, present, future. Revista de estudios sociales, 35(62), 10-15. https://doi. org/10.7440/res62.2017.02

Bondi, L., Davidson, J., \& Smith, M. (2005) Introduction: Geography's 'Emotional 
Turn'. En J. Davidson, L. Bondi, \& M. Smith, (Eds.). Emotional geographies. Hampshire UK y Burlington, VT: Ashgate. ISBN: 9780754643753.

Campos Medina, L., Silva Roquefort, R., \& Gaete Reyes, M. (2017). El rol de las emociones y los afectos en la producción del hábitat y el territorio. Revista invi, 32(91), 9-21. https://doi.org/10.4067/ s0718-83582017000300009

Cena, R. (2018). Bodies in context: the bodily/emotional dimensions as a category for understanding the world. Revista Latinoamericana de estudios sobre cuerpos, emociones y sociedad, 26(10), 8-10. Recuperado de http://relaces.com.ar/ fullissue/RELACES-N26.pdf

Colin, C. (2017). La nostalgia en la producción urbana: la defensa de barrios en Santiago de Chile. Revista invi, 32(91), 91-111. https://doi.org/10.4067/s071883582017000300091

Daughters, A. (2015). Solidarity and resistance on the Island of Llingua. Anthropology now, 7(1), 1- 10. https://doi. org/10.1080/19492901.2015.11728301

Daughters, A. (2016). Southern Chile's Archipelago of Chiloé: Shifting identities in a new economy. The journal of Latin American and Caribbean anthropology, 21(2), 317-335. https://doi.org/10.1111/ jlca.12199

Dallman, S., Ngo, M., Paris, P., \& Thien, D. (2013). Political ecology of emotion and sacred space: The Winnemem Wintu struggles with California water policy. Emotion, space and society, 6, 33-43. https://doi.org/10.1016/j. emospa.2011.10.006

Deleuze, G. \& Guattari, F. (2006). Mil mesetas. Capitalismo y esquizofrenia.
Valencia: Pre-texto. ISBN: 9788485081950. (Trabajo original publicado en 1980)

De Simone, R.L. (2015). Metamall: espacio urbano en la ciudad neoliberal chilena. Santiago: RIL editores. ISBN: 978-956-010174-7

Diaz, S. (2013-2014). Deleuze, Políticay Subjetividad. El parkour o la subjectivación lúdico-política de los cuerpos post-urbanos. Revista Latinoamericana de estudios sobre cuerpos, emociones y sociedad, 13(5), 8798. Recuperado de http://www.relaces.com. ar/index.php/relaces/article/view/237/193

Doshi, S. (2017). Embodied urban political ecology: five propositions. Area, 49(1), 125128. https://doi.org/10.1111/area.12293

Elmhirst, R., (2011). Introducing new feminist political ecologies. Geoforum, 42(2), 129-132. https://doi.org/10.1016/j. geoforum.2011.01.006

Goss, J. (1993). The 'magic of the mall': an analysis of form, function, and meaning in the contemporary retail built environment. Annals of the association of American geographers, 83(1), 18-47. Recuperado de https://www.jstor.org/stable/pdf/2569414. pdf

Graybill, J.K. (2013). Mapping an emotional topography of an ecological homeland: The case of Sakhalin Island, Russia. Emotional, space and society, 8, 39-50. https://doi. org/10.1016/j.emospa.2012.09.005

Haraway, D. (1991). Simians, cyborgs and women: The reinvention of nature. London: Free association books. ISBN 1853431397, 9781853431395

Harper, D. (2002). Talking about pictures: A case for photo elicitation. 
Visual studies, 17 (1), 13-26. https://doi. org/10.1080/14725860220137345

Harris, E.M., Martin, D.G., Polsky, C., Denhardt, L., \& Nehring, A. (2013). Beyond 'Lawn People': The role of emotions in suburban yard management practices. The professional geographer, 65(2), 345-361. https://doi.org/10.1080/00330124.2012.68 1586

Hayes-Conroy, J. \& Hayes-Conroy, A., (2013). Veggies and visceralities: A political ecology of food and feeling. Emotion, space and society, 6, 81-90. https://doi. org/10.1016/j.emospa.2011.11.003

Heynen, N. (2016). Urban political ecology II. Progress in human geography, 40(6), 839-845. https://doi. org/10.1177/0309132515617394

Hidalgo, C., Ther, F., Saavedra, G., \& Díaz, A. (2015). Affordances of landscapes and economic socio-spatial networks in the Quinchao archipelago, Chile: acontribution to landscape research and island studies. Island studies journal, 10(1), 49-70. Recuperado de http://dlc.dlib.indiana.edu/dlc/bitstream/ handle/10535/9761/ISJ-10-1-Hidalgo-etal $\% 5 \mathrm{~b} 1 \% 5 \mathrm{~d}$.pdf? sequence $=1 \&$ isAllowed $=\mathrm{y}$

Huiliñir-Curío, V. \& Zunino, H. M. (2017). Movilidad, utopías y lugares híbridos en Los Andes del Sur de Chile. Revista invi, 32(9), 141-160. https://doi.org/10.4067/s071883582017000300141

Jirón, P A., Imilian, W.A., \& Iturra, L. (2016). Relearning to travel in Santiago: the importance of mobile place-making and traveling know-how. Cultural geographies, 23(4), 599-614. https://doi. org/10.1177/1474474015622141

Kusenbach, M. (2003). Street phenomenology. Ethnography, 4(3), 455-485. https://doi.org/10.1177/146613810343007
Lindón, A. (2009). La construcción socioespacial de la ciudad: el sujeto cuerpo y el sujeto sentimiento. Revista Latinoamericana de estudios sobre cuerpos, emociones y sociedad, 1(1), 6-20. Recuperado de http://www.relaces.com.ar/ index.php/relaces/article/view/4/4

Lorimer, H. (2005). Cultural geography: the busyness of being more-thanrepresentational. Progress in human geography, 29(1), 83-94. https://doi. org/10.1191/0309132505ph531pr

Luna Zamora, R. \& Mantilla, L. (20172018). Desde la sociología de las emociones a la crítica de la Biopolítica. Revista Latinoamericana de estudios sobre cuerpos, emociones y sociedad, 25(9), 24-33. Recuperado de http://relaces.com.ar/index. php/relaces/article/view/565/391

Massumi, B. (2002). Parables for the virtual: Movement, affect, sensation. Durham and London: Duke University press.

McCormack, D. (2003). An event of geographical ethics in spaces of affect. Transactions of the institute of british geographers, 28(4), 488-507. https://doi. org/10.1111/j.0020-2754.2003.00106.x

Miller, J. (2018-2019). Embodied Architectural Geographies of Consumption and the Mall Paseo Chiloé Controversy in Southern Chile. Annals of the American association of geographers (in press)

Miller, J. (2018). No fish, no mall. Industrial fish produce new subjectivities in Southern Chile. Geoforum, 92, 125-133. https://doi. org/10.1016/j.geoforum.2018.04.005

Miller, J. (2015). The critical intimacies of walking in the Abasto shopping mall, Buenos Aires, Argentina. Social and cultural geography, 16(8), 869-887. https://doi.org./1 $0.1080 / 14649365.2015 .1026928$ 
Miller, J. (2014). Malls without stores (MwS): the affectual spaces of a Buenos Aires shopping mall. Transactions of the institute of British geographers, 39(1), 14-25. https://doi.org/10.1111/j.14755661.2012.00553.x

Mitchell, K. \& Elwood, S. (2012). Mapping children's politics: the promise of articulation and the limits of nonrepresentational theory. Environment and planning D: Society and space, 30 (5), 788-804. https://doi.org/10.1068/d9011

Moulian, T. (1997). Chile actual. Anatomía de un mito. Santiago: Lom-Archis. ISBN: 956-282-022-X

Moulian, T. (1998). El consumo me consume. Santiago: Lom. ISBN: 9799562820807

Nightingale, A. (2013). Fishing for nature: the politics of subjectivity and emotion in Scottish inshore fisheries management. Environment and planning A, 45(10), 2.3622.378. https://doi.org/10.1068/a45340

Ginga, L.N. \& Brizuela, F. (2017). Iniciativas escenográficas en Rosario: lo cool como tecnología de gobierno de subjectividades urbanas. Revista invi, 32 (91), 163-187. https://doi.org/10.4067/ s0718-83582017000300163

Rendueles, C., (2017). La gobernanza emocional en el capitalismo avanzado. Entre el nihilismo emotivista y el neocomunitarismo adaptativo. Revista de estudios sociales, 35(62), 82-88. https://doi. org/10.7440/res62.2017.08

Rocheleau, D., Thomas-Slayter, B., \& Wangari, E., (2013). Gender and environment. A feminist political ecology perspective. In D. Rocheleau, B. ThomasSlayter, \& E. Wangari (Eds.). Feminist political ecology: Global issues and local experience. (pp. 3-23). Routledge.
Recuperadodehttps://vtechworks.lib.vt.edu/ bitstream/handle/10919/70076/5011_ Rocheleau_Gender_and_Environment. pdf? sequence $=1$ \&isAllowed $=y$

Robbins, P. (2007). Lawn People. How grasses, weeds, and chemicals make us who we are. Philadelphia: Temple University press. ISBN-10: 9781592135790

Robbins, P. \& Krueger, R. (2000). Beyond bias? The promise and limits of Q method in human geography. The professional geographer, 52(4), 636-648. https://doi. org/10.1111/0033-0124.00252

Rose, G., Degen, M., \& Basdas, B. (2010). More on 'big things': building events and feelings. Transactions of the institute of British geographers, 35(3), 334-349. https:// doi.org/10.1111/j.1475-5661.2010.00388.x

Palmett Plata, O. (2015). Transformaciones conceptuales del paisaje urbano de Medellín. Investigaciones Geográficas, 50, 105-122. https://doi.org/10.5354/07195370.2015 .41184

Pile, S. (2010). Emotions and affect in recent human geography. Transactions of the Institute of British Geographers, 35(1), 5-20. https://doi.org/10.1111/j.14755661.2009.00368.x

Pincheira Torres, I. (2013). Entre el marketing empresarial y la política pública estatal: el gobierno de la felicidad en el neoliberalismo chileno. Revista Latinoamericana de estudios sobre cuerpos, emociones y sociedad, 11(5), 7-20. Recuperado de http://relaces.com. ar/index.php/relaces/article/view/217/153

Pyyry, N. (2018). From psychogeography to hanging-out-knowing: Situationist dérive in nonrepresentational urban research. Area. https://doi.org/10.1111/ area. 12466 
Robertt, P. (2009). Reestructuración industrial, social y de los cuerpos en el capitalism global. Revista Latinoamericana de estudios sobre cuerpos, emociones $y$ sociedad, 1(1), 21-29. Recuperado de http://relaces.com.ar/index.php/relaces/ article/view/6/1

Sarlo, B. (1994). Escenas de la vida podmoderna. Minneapolis: University of Minnesota press. ISBN: 950912222X, 9789509122222

Sarlo, B. (2009). La ciudad vista. Buenos Aires: Siglo veintiuno. ISBN: 978-987629-075-3

Sitrin, M. (2006). Horizontalism: Voices of popular power in Argentina. Oakland: AK press. ISBN 10: 1904859585. ISBN 13: 978-1904859581

Smith, M. (2013). Earthley passion(s): Essays towards an emotional ecology. Emotion, space and society, 6, 1-3. https:// doi.org/10.1016/j.emospa.2012.12.002
Thrift, M. (2008). Non-representational theory: space, politics, affect. London: Routledge. ISBN 10: 0415393213. ISBN 13: 978-0415393218

Tolia-Kelly, D.P. (2006). Affect-an ethnocentric encounter? Exploring the 'universalist' imperative of emotional/ affectual geographies. Area, 38(2), 213-217.

Woodward, K., Jones, J.P., \& Marston, S. (2012). The politics of autonomous space. Progressinhumangeography, 36(2), 204-224. https://doi.org/10.1177/0309132511432083

Wright, M.W. (2010). Geography and gender: feminism and a feeling of justice. Progress in human geography, 34(6), 818-27. https://doi. org/10.1177/0309132510362931

Wylie, J. (2005). A single day's walking: narrating self and landscape on the South West Coast Path. Transactions of the institute of British geographers, 30(2), 234-247. https://doi.org/10.1111/j.14755661.2005.00163.x 